\title{
Short $\gamma$-ray bursts and gravitational waves from dynamically formed merging binaries
}

\author{
D. Guetta and L. Stella
}

\author{
Osservatorio astronomico di Roma, v. Frascati 33, 00040 Monte Porzio Catone, Italy \\ e-mail: dafne@arcetri.astro.it
}

Received 1 July 2008 / Accepted 26 October 2008

\begin{abstract}
Merging binary systems consisting of two collapsed objects are among the most promising sources of high frequency gravitational wave, GW, signals for ground based interferometers. Double neutron star or black hole/neutron star mergers are also believed to give rise to short hard bursts (SHBs) a subclass of gamma ray bursts. SHBs might thus provide a powerful way to infer the merger rate of two-collapsed object binaries. Under the hypothesis that most SHBs originate from double neutron star or black hole/neutron star mergers, we outline here a method to estimate the incidence of merging events from dynamically formed binaries in globular clusters and infer the corresponding GW event rate that can be detected with Advanced LIGO/Virgo. In particular, a sizeable fraction of detectable GW events is expected to be coincident with SHBs. The beaming and redshift distribution of SHBs are reassessed and their luminosity function constrained by using the results from recent SHB observations. We confirm that a substantial fraction of SHBs occur at low redshifts, where the merging of systems formed in globular clusters through dynamical interactions is expected.
\end{abstract}

Key words. gamma rays: bursts - stars: binaries: general - stars: neutron - gravitational waves

\section{Introduction}

Merging binary systems containing two collapsed objects, i.e. a double neutron star (DNS), a stellar-mass black hole and neutron star (BH-NS) or two stellar-mass black hole (BH-BH), are powerful gravitational wave $(\mathrm{GW})$ sources with frequencies from hundreds to over a thousand $\mathrm{Hz}$. They are considered among the most promising GW sources for ground-based interferometers, of the current and future generation, such as LIGO, Virgo and their advanced versions. BH-NS and, especially, BH-BH mergers emit more powerful and lower frequency GWs than DNS mergers, where the sensitivity of LIGO and Virgo detectors is highest: therefore they can be detected up to greater distances. The horizon of first generation LIGO and Virgo for DNSs, BHNS and BH-BH mergers is $\sim 20,43$ and $100 \mathrm{Mpc}$, respectively, while Advanced LIGO/Virgo class interferometers should detect them out to a distance of $\sim 300,650$ and $1600 \mathrm{Mpc}$ (for a review see Cutler \& Thorne 2002).

The rate of detectable merging events has been estimated based on the observed galactic population of DNS binaries containing a radio pulsar (Phinney 1991; Narayan et al. 1991; Kalogera et al. 2001; Burgay et al. 2003). The best estimate of the DNS merger rate in the Galaxy is presently $\sim 80_{-60}^{+200} / \mathrm{Myr}$, converting to $800_{-600}^{+2000} \mathrm{Gpc}^{-3} \mathrm{yr}^{-1}$ for a galaxy number density of $10^{-2} \mathrm{Mpc}^{-3}$ (Kalogera et al. 2004). Population synthesis studies of binary systems give results consistent with the above rate (Perna \& Belczynski 2002; Belczynski et al. 2002, 2007). GW signals from DNS mergers are expected at a rate of one in $\sim 10-150$ years with Virgo and LIGO and one every 1-15 days with Advanced LIGO/Virgo class interferometers. The BH-NS and BH-BH merger rates in the Galaxy are highly uncertain. Belczynski et al. (2007) estimate $\sim 1 \%$ and $\sim 0.1 \%$ of the DNS merger rate, respectively, implying that $\mathrm{BH}-\mathrm{NS}$ and $\mathrm{BH}-\mathrm{BH}$ mergers contribute marginally to the $\mathrm{GW}$ event rate, despite the greater distance up to which they can be detected.

DNSs and NS-BH merging events also provide one of the leading models for short hard gamma ray bursts (SHBs), i.e. bursts of $\gamma$-rays that last $<2 \mathrm{~s}$ and take place at cosmological distances (Goodman 1986; Paczyński 1986; Eichler et al. 1989; Narayan et al. 1992). As with the collapsar scenario for long Gamma Ray Bursts, GRBs (Woosley \& Mac Fadyen 1999), such models envisage the formation of a black hole surrounded by a torus of matter at nuclear density that is rapidly accreted and provides the primary source of energy for the burst. For this reason it is believed that $\mathrm{BH}-\mathrm{BH}$ mergers are not among the progenitors of SHBs. SHBs comprise about $1 / 4$ and $1 / 10$ of the CGRO/BATSE and Swift/BAT GRB samples. The X-ray and optical afterglows of SHBs have been observed only recently, leading to the first identifications and redshift determinations of SHB host galaxies (see e.g. Berger et al. 2007a and references therein). Out of the $\sim 30$ SHBs in the current Swift and HETE 2 sample about half displayed an optical afterglow, 11 have redshift, and a few show evidence of beaming in their optical afterglow light curve (Fox et al. 2005; Soderberg et al. 2006). The association of some SHBs with galaxies characterized by a very low star formation rate (SFR, of the order of $\lesssim 0.1 M_{\odot} \mathrm{yr}^{-1}$ ) indicates a long timedelay between the progenitors' collapse and the time at which these SHBs occur.

It has long been known that DNSs and BH-NS systems (hereafter NS-NS/BH systems), can form from massive binaries surviving two gravitational collapses (we term these "primordial" NS-NS/BH systems). Population synthesis calculations show that primordial NS-NS/BH systems merge after a relatively short time ( $\tau \approx 2 \mathrm{Gyr}$ on average), implying that the redshift-distribution of SHBs should follow closely the SFR history of the universe (e.g. Bloom et al. 1999; Belczynski et al. 2002, 2006). Alternatively NS-NS/BH systems can form through 
dynamical interactions in the cores of globular clusters (GCs). The dominant process comprises two stages: first an isolated NS (or $\mathrm{BH}$ ) in the cluster captures a non-degenerate star during a close encounter, thus forming a binary containing a collapsed object. This binary then undergoes an exchange interaction with another collapsed object, leading to the ejection of the non-degenerate star and formation of a NS-NS/BH binary (Verbunt \& Hut 1987; Efremov 2000). Grindlay et al. (2006) estimate that $\sim 10-30 \%$ of all DNS mergers may stem from such dynamically formed systems. Dynamical interactions take place efficiently at high stellar densities, which, in turn, are highest in the core of globular clusters that have undergone core collapse. Therefore, the delay between the formation of the collapsed objects and merging of dynamically-formed NS-NS/BH systems is dominated by the time until core-collapse, which is typically comparable to the Hubble time. In their simulations Sadowski et al. (2008) found that merging $\mathrm{BH}-\mathrm{BH}$ binaries form rather efficiently in dense clusters, but failed to find any merging of NS-NS/BH binaries.

If NS-NS/BH mergers are among the progenitors of SHBs, the SHB rate can be used as an alternative way of constraining the DNS merger rate (Guetta \& Piran 2005, 2006 (GP05, GP06), Nakar et al. 2006). Hopman et al. (2006) (H06) showed that dynamically formed DNSs give rise to more numerous low- $z$ bursts than would be expected if SHB closely followed the SFR, as in the case of primordial DNSs. The z-distribution of dynamically formed DNSs provides a better match to observed redshifts of SHBs. This result was recently confirmed by Salvaterra et al. (2008), who also conclude that SHBs may well originate from both classes of DNSs. GP05, GP06 and H06 fitted the peak flux distribution of SHBs detected by BATSE to derive their formation rate and luminosity function (LF), in both the dynamicallyformed and primordial DNS scenarios, while Nakar et al. (2006) and GP06 studied the prospects for detecting GW signals from SHBs from primordial DNSs.

In this paper, based on the results from recent SHB observations, we first reassess the beaming, redshift distribution and LF of SHBs. Under the hypothesis that NS-NS/BH mergers give rise to most $\mathrm{SHBs}$, we then present a method to estimate the incidence of merging events from dynamically formed binaries in globular clusters and infer the corresponding GW event rate that could be detected with Advanced LIGO/Virgo class interferometers.

\section{Luminosity function and rate evolution of SHBs}

For primordial NS-NS/BH systems, the intrinsic SHB rate is given by the convolution of the formation rate of NS-NS/BH binaries (which is assumed to follow the SFR with negligible delay ) with the distribution of the merging time delays, $P_{\mathrm{pr}}(\tau)$, where $\tau=\tau_{\mathrm{GW}}$ is the time over which GW losses bring a binary to its pre-merging stage. We adopt $P_{\mathrm{pr}}(\tau) \sim 1 / \tau$ in agreement with the $\tau$-values of DNS radio pulsar binaries and population synthesis calculations (Champion et al. 2004; Belczynski et al. 2007).

The SHB rate from primordial DNSs is given by

$R_{\mathrm{SHB}}(z) \propto \int_{0}^{t(z)} \mathrm{d} \tau R_{\mathrm{SF} 2}(t-\tau) P_{\mathrm{pr}}(\tau)$,

where $R_{\mathrm{SF} 2}$ refers to the "SF2" model for the SFR in Porciani \& Madau (2001).

For a dynamically formed NS-NS/BH binary, $\tau=\tau_{\mathrm{CC}}+\tau_{\mathrm{GW}}$, where the delay time $\tau_{\mathrm{CC}} \gg \tau_{\mathrm{GW}}$ represents the elapsed time between the birth of NSs and BHs in GCs and the dynamical formation of NS-NS/BH systems following core collapse.
Table 1. Model parameters and local rates.

\begin{tabular}{lllll}
\hline \hline DNSs (BH-NS) & $\begin{array}{l}L^{*} \\
{\left[10^{51} \mathrm{erg} \mathrm{s}^{-1}\right]}\end{array}$ & $\alpha$ & $\beta$ & $\begin{array}{l}R_{0} \text { Rate }(z=0) \\
\mathrm{Gpc}^{-3} \mathrm{yr}^{-1}\end{array}$ \\
\hline Primordial & 2 & 0.6 & 2 & 1.3 \\
Dynamically formed & 0.8 & 0.8 & 2 & 4.0 \\
\hline
\end{tabular}

According to H06, the distribution of dynamically formed NSNS/BH binaries $P_{\text {dyn }}(\tau)$ (which replaces $P_{\mathrm{pr}}(\tau)$ in Eq. (1)) increases for increasing time delays. We assume that the formation rate of GCs is proportional to the total $\mathrm{SFR}^{1}$. We derive an average delay of $\bar{\tau} \approx 2$ and $\approx 6 \mathrm{Gyr}$, for primordial and dynamically formed NS-NS/BH systems, respectively (for the latter we considered only values of $\tau$ shorter than the Hubble time).

\subsection{The SHB luminosity function}

In this section we summarize the method adopted by GP06 and H06 to estimate the SHBs' LF and we derive the local SHB rates independently for primordial and dynamically formed NSNS/BH binaries. We use here the same sample of 194 SHBs detected by BATSE as in Paciesas et al. (1999). GP06 and H06 modelled the "isotropic-equivalent" LF (i.e. uncorrected for beaming), with a broken power law extending from $L^{*} / \Delta_{1}$ to $L^{*} \Delta_{2}$ and with break at $L^{*}$ (with $\Delta_{1}=\Delta_{2}=100$ ), i.e.

$\Phi_{o}(L) \mathrm{d} \log L=C_{0} \mathrm{~d} \log L \begin{cases}\left(L / L^{*}\right)^{-\alpha} ; & L^{*} / \Delta_{1}<L<L^{*} \\ \left(L / L^{*}\right)^{-\beta} ; & L^{*}<L<\Delta_{2} L^{*}\end{cases}$

( $C_{0}$ is a normalization constant). The best fit LF was derived by using the SHB rate $R_{\mathrm{SHB}}(z)$ for the two formation scenarios (Eq. (1)). Using these "best fit" LFs these authors calculated the predicted redshift distribution in the two cases and compared it with the present sample of SHB redshifts (see Sect 2.2). By using the same procedure, we rederived the best fit parameters for the LF and determined for the first time the local event rate, $R_{0}$ (see Table 1), independently in the two scenarios. We find that, by virtue of their longer $\tau$, dynamically formed systems would have to merge at a $\sim 3$ times higher rate than primordial systems in the local universe.

The results in Table 1 are virtually insensitive to an increase of $\Delta_{2}$, whereas $L_{\min }=L^{*} / \Delta_{1}$ cannot be increased by more than a factor of 10 without excluding the least luminous SHB detected so far (GRB050509B, Gehrels et al. 2005). By decreasing $L_{\min }=L^{*} / \Delta_{1}$, the local SHB rate increases approximately as $R_{0} \propto\left(L_{\min }\right)^{\alpha}$ (Nakar 2006). Objects with luminosity $L_{\min }$ observed by CGRO/BATSE or $S$ wift/BAT at a limiting flux of $F_{\text {lim }}$ are detectable up to a maximum redshift of $z_{\max }$ which decreases with decreasing $L_{\min }$ (for Swift/BAT $F_{\text {lim }}$ is $\sim 1 \mathrm{ph} \mathrm{cm}^{-2} \mathrm{~s}^{-1}$; Sakamoto et al. 2008). Therefore SHBs with very low luminosity are above the limiting sensitivity of BATSE and BAT only in a fairly small volume, where they appear rarely. Current data do not constrain this part of the LF and the number of very weak SHBs (if any) remains unknown. It cannot be excluded that the volume afforded by the limiting sensitivity of BATSE and BAT is close to that required in order to sample the low-luminosity end of the LF of SHBs over the satellites' lifetime. However this appears contrived and the low-luminosity end of the SHB LF might well extend below the BATSE and

\footnotetext{
1 We note that Salvaterra et al. (2008) considered two alternative GC formation rates and found that these produce very similar results to those obtained by assuming that GC formation follows the SFR.
} 
BAT sensitivity. We note in passing that the presently-known LF of long GRBs extends over more than 4 decades (see e.g. Liang et al. 2007; Guetta \& Della Valle 2007). In Sect. 3 we discuss further the implications of a SBH LF that extends below the currently-estimated value.

There exists evidence that SHBs are beamed in a relatively small solid angle. Similarly to the case of long GRBs, the jet opening angle is inferred from the steepening of the optical afterglow light curve. Fox et al. (2005) found a beaming factor of $f_{\mathrm{b}}^{-1} \sim 50$ for GRB 050709 and GRB 050724 (with $f_{\mathrm{b}}$ the fraction of the $4 \pi$ solid angle within which the GRB is emitted). Soderberg et al. (2006) inferred $f_{\mathrm{b}}^{-1} \sim 130$ for GRB 051221A. We adopt a fiducial value of $f_{\mathrm{b}}^{-1} \sim 100$ and derive a beaming-corrected rate of $\rho_{0}=f_{\mathrm{b}}^{-1} R_{0} \sim 130\left(f_{\mathrm{b}}^{-1} / 100\right)$ and $\sim 400\left(f_{\mathrm{b}}^{-1} / 100\right) \mathrm{Gpc}^{-3} \mathrm{yr}^{-1}$ for SHBs originating in primordial and dynamically formed binaries, respectively. The former estimate compares well with the lower end of the range estimated by Belczynski et al. (2002) and Kalogera et al. (2004) for primordial DNSs (200-2800 $\left.\mathrm{Gpc}^{-3} \mathrm{yr}^{-1}\right)$.

\subsection{The observed $z$ distribution of SHBs}

In order to infer the redshift distribution of SHBs, we use the entire sample of presently known SHBs with redshift determination, i.e. the sample of events in Table 2 of Salvaterra et al. (2008) plus GRB 071227, GRB 070429B and GRB 070714 at $z=0.384, z=0.902$ and $z=0.922$, respectively (D'Avanzo et al. 2007; Berger et al. 2007b; D'Avanzo et al. 2008; Cenko et al. 2008). We excluded two of the bursts (GRB 061006, GRB 061201) from the sample of Salvaterra et al. (2008) because uncertainties in the redshift determination ${ }^{2}$. We have verified that re-inserting these bursts in our sample does not significantly affect any of our results.

In Fig. 1 we compare the cumulative distribution of the observed redshifts with the expected cumulative $z$-distribution given by

$N_{\exp }(z)=\frac{R_{\mathrm{SHB}}(z)}{1+z} \frac{\mathrm{d} V(z)}{\mathrm{d} z} \int_{L_{m}}^{L_{\max }} \Phi_{o}(L) \mathrm{d} \log L$,

where $L_{\max }=\Delta_{2} L^{*}=100 L^{*}$ and $L_{m}=\max \left(L_{\mathrm{F}}, L^{*} / \Delta_{1}\right)$, with $L_{\mathrm{F}}$ the luminosity at $z$ corresponding to $F_{\text {lim }}$. This is done in turn for the primordial and dynamical formation scenarios, by using the parameters given in Table 1 and $\Delta_{1}=100$ (see Fig. 1). A Kolmogorov-Smirnov (KS) test gives a probability of 0.04 and 0.2 that the observed distribution is drawn from the distribution expected for SHBs from primordial and dynamically formed NS-NS/BH binaries, respectively. These results are nearly insensitive to increasing values of $\Delta_{1}$ (see also in Sect. 2.1).

We then considered the cumulative $z$-distribution that results from a combination of primordial and dynamically formed NS$\mathrm{NS} / \mathrm{BH}$ systems. By varying their relative incidence the closest match with the observed $z$-distribution of SHBs was found for a $\sim 60 \%$ contribution from dynamically formed NS-NS/BH binaries (see the dot-dashed line in Fig. 1). In order obtain a rough lower limit on the incidence of dynamically formed NS-NS/BH systems we decreased their contribution until a KS probability of 0.1 was reached: this gives a $\sim 10 \%$ incidence of such systems. We note that the corresponding local SHB rate is $R_{0} \sim 2.9$ and $1.6 \mathrm{Gpc}^{-3} \mathrm{yr}^{-1}$ for a $60 \%$ and $10 \%$ fraction of dynamically formed mergers, respectively.

\footnotetext{
${ }^{2}$ However we note that D'Avanzo et al. (2008) have recently confirmed the redshift of GRB 061006.
}

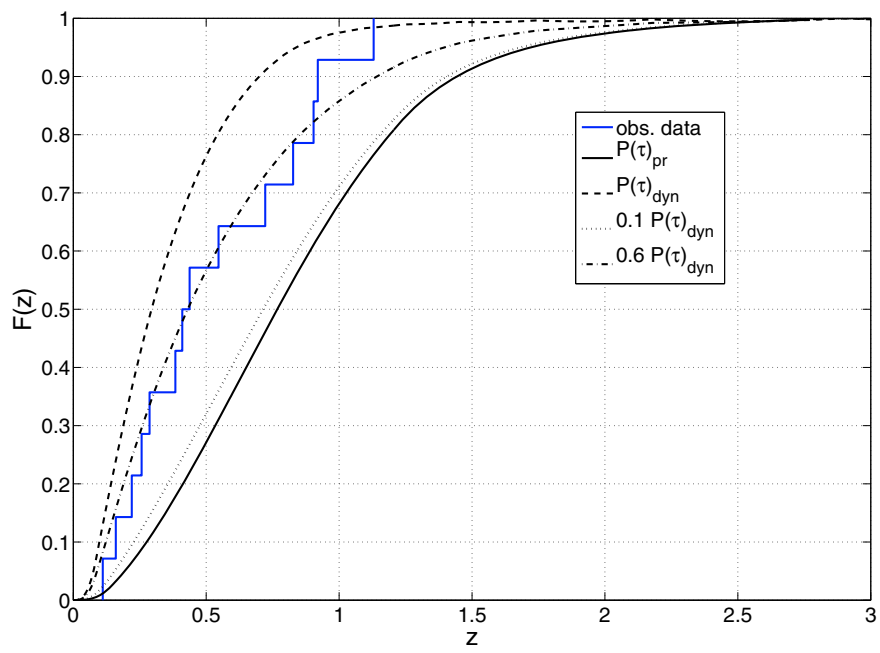

Fig. 1. Observed (histogram) and expected (curves) cumulative $z$-distribution functions for the different time-delay probabilities expected in the two scenarios. Model distributions are shown for different combination of primordial and dynamically formed NS-NS/BH systems.

Of course this incidence can be derived only approximately, considering the small sample of SHBs with redshift determinations known so far. We emphasise that the relative contribution of the two populations of merging binaries is determined primarily by the observed redshift distribution of $\mathrm{SHBs}^{3}$, which can be constrained at present only through the 11 SHBs with known redshift.

The above estimate compares well with the fraction $(10-30 \%)$ of SHBs produced in GCs, as derived by Grindlay et al. (2006). We conclude that the present sample of SHBs with redshift determination favors a bimodal origin of SHBs, with high- $z$ bursts resulting primarily from the merging of primordial NS-NS/BH systems and low- $z$ bursts produced mainly by dynamically-formed systems, as first proposed by Salvaterra et al. (2008). The contribution from the latter systems is unlikely to be $<10 \%$. This conclusion might be altered if there existed a large population of high redshift (and thus mainly primordial) SHBs that has so far remained unidentified (Berger et al. 2007a; O'Shaughnessy et al. 2008).

On the other hand the association of a SHB with a GC would confirm unambiguously the dynamical formation scenario. The large offset of some SHBs from their host galaxy is consistent with that of $\mathrm{GCs}^{4}$, but direct evidence for this is still lacking. This issue may also be addressed statistically by considering that, even though GCs are present in galaxies of virtually all types ${ }^{5}$, most of them are in early type galaxies. Therefore SHBs from dynamically formed binaries, besides having on average lower redshifts than those from primordial binaries, should be associated preferentially with early type galaxies. Since our sample largely overlaps with that of Salvaterra et al. (2008), we obtain similar results to theirs in this respect, with 3 in 3 SHBs associated with early type galaxies at $z<0.3$, and 1 in 8 SBHs in association with early type galaxies at $z>0.3$. While these results are in broad agreement with a bimodal origin of NS-NS/BH

\footnotetext{
3 The other uncertainties in the expected cumulative distribution, see Eq. (3), play a negligible role here.

4 See Gehrels et al. (2005) for the case of GRB050509b and Bloom et al. (2006) for GRB060502b.

${ }^{5}$ Except perhaps dwarf galaxies with $M_{V}>-13$.
} 
systems giving rise to SHBs, the low numbers involved prevent us from reaching any firm conclusion.

The beaming-corrected SHB rate scales as

$\rho_{0} \simeq 100\left(f_{\mathrm{b}}^{-1} / 100\right)\left(\Delta_{1} / 100\right)^{\alpha} R_{0}$.

If dynamically formed DNS mergers represent $10 \%$ of the total SHB local rate ${ }^{6},\left(f_{\mathrm{b}}^{-1} / 100\right)\left(\Delta_{1} / 100\right)^{\alpha} \sim 0.1$ would be required for Eq. (4) to match the approximate merger inferred by Phinney et al. (1991) for GC DNSs $\left(\rho_{\text {DNS }} \sim 3 \mathrm{Gpc}^{-3} \mathrm{yr}^{-1}\right)$. This indicates that the rough estimate by Phinney et al. (1991) is about an order of magnitude lower than the beaming-corrected rate derived from SHB observations, a difference that we do not regard as crucial at this stage, considering all the uncertainties.

\section{Prospects for detection of gravitational waves}

Within the NS-NS/BH binary interpretation, the local rate of SHBs has clear implications for the number of merging events that can be detected with present and future ground-based GW interferometers. Based on the results in Sect. 2, we estimate here the number of detectable GW events expected for Advanced LIGO/Virgo class interferometers due to SHBs originating from NS-NS/BH mergers,

$$
\begin{aligned}
N_{\mathrm{GW}} \sim & \eta \rho_{0}\left[\left(1-g_{\mathrm{B}}\right) V_{\mathrm{DNS}}+g_{\mathrm{B}} V_{\mathrm{BHNS}}\right] \\
\simeq & 45 \eta\left(\frac{f_{b}^{-1}}{100}\right)\left(\frac{R_{0}}{4 \mathrm{Gpc}^{-3} \mathrm{yr}^{-1}}\right)\left(\frac{\Delta_{1}}{100}\right)^{\alpha} \\
& \times\left[\left(1-g_{\mathrm{B}}\right)+g_{\mathrm{B}} \frac{V_{\mathrm{BHNS}}}{V_{\mathrm{DNSs}}}\right] \mathrm{yr}^{-1},
\end{aligned}
$$

where $g_{\mathrm{B}}$ is the incidence of BH-NS systems among NS-NS/BH progenitors of SBHs, $V_{\mathrm{DNS}}$ and $V_{\mathrm{BHNS}}$ are the volumes corresponding to the horizon of GW interferometers for DNSs and BH-NS mergers respectively; the ratio between these volumes is $V_{\mathrm{BHNS}} / V_{\mathrm{DNS}} \sim 10$. Here $\eta=1$ for Advanced LIGO/Virgo and $\eta=3 \times 10^{-4}$ for LIGO/Virgo take into account the different volumes sampled by the two instrument classes (see e.g. Cutler \& Thorne 2002). While it is estimated that only $g_{\mathrm{B}} \sim 0.01$ of primordial NS-NS/BH systems are BH-NS binaries (Belczynski et al. 2007), the fraction of such systems in GCs, though presently unknown, might well be higher, their main formation channel being the exchange interaction of a binary containing a NS and a cluster star with an isolated BH (Devecchi et al. 2007). Dynamical star cluster simulations point to a fairly large population of $\mathrm{BH}-\mathrm{BH}$ binaries in $\mathrm{GCs}$, but have not yet provided estimates of $g_{\mathrm{B}}$ (O'Leary et al. 2006). For instance for the globular cluster parameters adopted by Sadowski et al. (2008) NS-NS/BH binaries are expected to form at a very slow pace, and it is not surprising that no such systems were produced in those simulations.

We note that as long as $g_{\mathrm{B}} \gtrsim 0.15$ the rate of detectable GW events from BH-NS binaries will be higher than that from DNSs (see Eq. (5)). We assume $g_{\mathrm{B}}=0.5$ (and the other fiducial values in Eq. (5)) in the estimates of $N_{\mathrm{GW}}$ given below, and report also in parentheses the values corresponding to $g_{\mathrm{B}} \sim 0$ and 1 . For Advanced LIGO/Virgo we find that events from primordial and dynamically formed NS-NS/BH binaries are expected at a rate of $\sim 14 \mathrm{yr}^{-1}$ and $\sim 248 \mathrm{yr}^{-1}(45,450)$, respectively. For $g_{\mathrm{B}}=0.5$ the latter event rate is dominated by merging BH-NS binaries formed in GCs $\left(\sim 226 \mathrm{yr}^{-1}\right)$ and is close to the upper end of the range estimated by Kalogera et al. (2006) for primordial DNSs.

\footnotetext{
${ }^{6}$ Note that the contribution from BH-NS mergers is not included.
}

For present generation interferometers Eq. (5) gives 1 event in 238 years and 1 event in 13 years $(74,7$ years) from primordial and dynamically formed systems, respectively.

There are presently substantial uncertainties in the values of the parameters in Eq. (5) and therefore a precise estimate of the expected number of detectable GW event cannot be made yet. However it should be possible in the near future to constrain more tightly the uncertain parameters in Eq. (5). Concerning the beaming factor, a few additional detailed studies of the optical afterglow of SHBs with currently available instrumentation will allow a more precise determination of $f_{\mathrm{b}}^{-1}$. Taken at face value, the present estimates range over a factor of $\sim 2.6$. The local SHB event rate $R_{0}$, while constrained by the large sample of SHBs revealed by BATSE, can vary by up to a factor of $\sim 1.8$ depending on the redshift distribution and incidence of dynamically formed SHBs (see Sects. 2.1 and 2.2). The latter is presently determined through 11 SHBs, but the sample of SHBs with secure redshift is steadily increasing and should triple by the end of the Swift mission. A more accurate determination of the lower end of the SHB luminosity function will likely require more sensitive GRB detectors than currently available; however, as we discussed in Sect. 2.1 the lower limit $L_{\text {min }}$ will likely decrease (i.e. $\Delta_{1}$ will increase) resulting in a higher expected rate of detectable GW events. The incidence of NS-BH systems among dynamically formed SHB progenitors $g_{\mathrm{B}}$ can be determined through more extensive dynamical GC simulations. The whole range of allowed values of $g_{\mathrm{B}}($ i.e. $0 \div 1)$ translates into a factor of $\sim 10$ uncertainty in the number of GW events in Eq. (5).

Our treatment assumes that SHBs with similar properties are produced both by DNS and BH-NS mergers. From the point of view of the models, this appears to be a reasonable ansatz as the torus of matter at nuclear densities is expected to originate from the lightest collapsed object in the system and provide the main source of energy for the SHB. Moreover, observations have not yet shown clear evidence that SHBs comprise different subclasses. Alternatively, if all SHBs detected so far came from DNSs, the total GW event rate from dynamically formed DNS and BH-NS mergers would be higher (unless $g_{\mathrm{B}}=1$ holds). On the more pessimistic side, if a fraction $h$ of SHBs were due to an entirely different phenomenon (e.g. giant flares from soft gamma repeaters, Hurley et al. 2005) all the rates given above would be decreased by a factor $\sim(1-h)$.

According to Sadowski et al. (2008), the rate of $\mathrm{BH}-\mathrm{BH}$ mergers formed in globular clusters is high and can give rise to $0.01-1$ event per year in LIGO and 25-3000 events per year in Advanced LIGO, a major uncertainty being the initial stellar mass fraction in dense clusters. Other authors envisage substantially lower rates (Portegies Zwart \& McMillan 2000, O’Leary et al. 2006). Once the ratio between NS-NS/BH and BH-BH binaries in globular clusters will be better known from simulations, the merging rate of dynamically formed NS-NS/BH binaries inferred from SHBs can provide an independent normalization for the $\mathrm{BH}-\mathrm{BH}$ merger rate.

We note that an interferometer's horizon would increase by a factor of $\sim 2.4$ for a GW detection coinciding with a SHB detection (Cutler \& Thorne 2002). This is because of the higher sensitivity afforded by having independent knowledge of the time of occurrence and sky position of the event. The above factor also takes into account that, if SHBs are beamed along the binary's angular momentum axis, then the SHBs that are detected at the earth are also the ones whose orientation angle maximises the $\mathrm{GW}$ signal. The larger horizon translates into a factor of $\sim 15$ increase in both $V_{\text {DNS }}$ and $V_{\text {BHNS }}$. Considering that only a fraction $f_{\mathrm{b}} \sim 0.01$ of the SHBs are beamed toward us, the coincident 
event rate would be $\sim 0.15\left(f_{\mathrm{b}}^{-1} / 100\right)$ of the rates derived from Eq. (5).

\section{Conclusions}

In this paper we have presented a simple method for inferring the merging rate and corresponding rate of detectable GW signals from coalescing NS-NS/BH binaries. The method is based on the assumption that at least a sizable fraction of SHBs originates in the merging of such binaries and exploits the most recent results from observations to infer the luminosity function, redshift distribution and degree of beaming of SHBs. We find further evidence in favor of a bimodal origin of SHB progenitors, with the merging of primordial binaries dominating at high redshift, whereas at lower redshift at least $\sim 10 \%$ (and probably about half) of the events arise from coalescing binaries formed dynamically in GCs. We have shown that the latter events make the expected local merging rate higher.

The accuracy of the GW event rate that can be estimated at present is hampered by our current knowledge of the parameters in Eq. (5). Maximum uncertainties in the SHB beaming factor $\left(f_{\mathrm{b}}\right)$ and local rate $\left(R_{0}\right)$ add up to a factor of $\sim 5$, whereas the ignorance of the incidence $g_{\mathrm{B}}$ of BH-NS binaries among dynamically formed systems adds a factor of 10 uncertainty. This results in an increase of total uncertainty by a factor of $\sim 50$ in the detectable GW event rate in Eq. (5). Though large, this is not far from the uncertainties of other estimates of GW events in literature. For instance Kalogera et al. (2004) estimate the galactic double neutron star merger rate with an uncertainty of a factor of 14 . Similarly, the results of the dynamical simulations of Sadowski et al. (2008) for BH-BH mergers are affected by a 1.5 decade wide uncertainty in the initial mass fraction of globular clusters. We expect that further SHB observations in the Swift era will lead to a more accurate determination of $f_{\mathrm{b}}$ and $R_{0}$, while more advanced dynamical globular cluster simulations will decrease the range of allowed values of $g_{\mathrm{B}}$. Progress in this area might take place at a sufficiently fast pace that our method can yield accurate predictions before GW events from coalescing binaries are detected in large numbers.

We have shown that a sizeable fraction of detectable GW events is expected to be coincident with SHBs (depending on the SHB beaming factor): this provides a new interesting perspective for the Advanced LIGO/Virgo era. We conclude that SHBs can provide a powerful means of inferring the $\mathrm{GW}$ event rate from coalescing binaries containing two collapsed objects.
Acknowledgements. We acknowledge useful discussions with Monica Colpi, Livia Origlia and Andrea Possenti. This work was partially supported through ASI/INAF contracts ASI/I/R/039/04 and ASI/I/R/023/05.

\section{References}

Belczynski, K., Kalogera, V., \& Bulik, T. 2002, ApJ, 572, 407 Belczynski, K., Perna, R., Bulik, T., et al. 2006, ApJ, 648, 1110 Belczynski, K., Perna, R., Bulik, T., et al. 2007, ApJ, 662, 504 Berger, E., Fox, D. B., Price, P. A., et al. 2007a, ApJ, 664, 1000 Berger, E., Morrell, N., \& Roth, M. 2007b, GCN, 7154

Bloom, J. S., Sigurdsson, S., \& Pols, O. R. 1999, MNRAS, 305, 763 Bloom, J. S., Perley, D., Kocevski, D., et al. 2006, GCN, 5238

Burgay, M., D'Amico, N., Possenti, A., et al. 2003, Nature, 426, 531 Cenko, B. S., et al. 2008, [arXiv:0802.0874]

Champion, D. J., Lorimer, D. R., McLaughlin, M. A., et al. 2004, MNRAS, 350, L61

Cutler, C., \& Thorne, K. S. 2002, Proc. of GR16, [arXiv:gr-qc/0204090] D'avanzo, P., Fiore, F., Piranomonte, S., et al. 2007, GCN, 7152

D'avanzo, P. et al. 2008, A\&A, submitted

Efremov, Yu. N. 2000, Astron. Lett. 26, 558

Eichler, D., Livio, M., Piran, T., \& Schramm, D. N. 1989, Nature, 340, 126

Fox, D. B., Frail, D. A., Price, P. A., et al. 2005, Nature, 437, 845

Gehrels, N., Sarazin, C. L., O'Brien, P. T., et al. 2005, Nature, 437, 851

Goodman, J. 1986, ApJ, 308, L47

Grindlay, J., Portegies Zwart, S. F., \& McMillan, S. 2006, Nat. Phys. 2, 116

Guetta, D., \& Della Valle, M. 2007, ApJ, 657, L73

Guetta, D., \& Piran, T. 2005, A\&A, 435, 421 (GP05)

Guetta, D., \& Piran, T. 2006, A\&A, 453, 823 (GP06)

Guetta, D., \& Piran, T. 2007, JCAP, 07, 3

Hopman, C., Guetta, D., Waxman, E. \& Portegies Zwart, S. F. 2006, ApJ, 643, L91 (H06)

Hurley, K., Boggs, S. E., Smith, D. M., et al. 2005, Nature, 434, 1098

Kalogera, V., Narayan, R., Spergel, D. N., \& Taylor, J. H. 2001, ApJ, 556, 340

Kalogera, V., Kim, C., Lorimer, D. R., et al. 2004, ApJ, 601, L179

Liang, E. Zhang, B., Virgili, F., \& Dai, Z. G. 2007, ApJ, 662, 1111

Nakar, E., Gal-Yam, A., \& Fox, D. 2006, ApJ, 650, 281

Narayan, R., Piran, T. \& Shemi, A. 1991, ApJ, 379, L17

Narayan, R., Paczynski, B., \& Piran, T. 1992, ApJ, 395, L83

Oechslin, R., \& Janka, H. T. 2007, Phys. Rev. Lett., 99, 121102

O'Leary, R. M., Rasio, F. A. Fregeau, J. M. Ivanova N., \& O'Shaighnessy, R. 2006, ApJ, 637, 937

O’Shaughnessy, R., Belczynski, K., \& Kalogera, V. 2008, ApJ, 675, 566

Paciesas, W., Meegan, C. A., Pendleton, G. N. et al. 1999, ApJS, 122, 465

Paczyński, B. 1986, ApJ, 308, L43

Perna, R., \& Belczynski, K. 2002, ApJ, 570, 252

Phinney, E. S. 1991, ApJ, 380, L17

Portegies Zwart, S., \& McMillan, S. L. W. 2000, ApJ 528, L17

Sadowski, A., Belczynski, K., Bulik, T., et al. 2008, ApJ, 676, 1162

Sakamoto, T., Barthelmy, S. D., Barbier, L., et al. 2008, ApJS, 175, 179

Salvaterra, R., Cerutti, A., Chincarini, G., et al. 2008, MNRAS, 388, L6

Sigurdsson, S. Phinney, E. S. 1995, ApJS, 99, 609

Soderberg, A. 2006, ApJ, 650, 261

Verbunt, F., \& Hut, P. 1987, Proc. IAU Symp., 125, 187

Woosley, S., \& MacFadyen, A. I. 1999, A\&AS, 138, 499 